\title{
Reformen im Parteien- und Ländervergleich: Gelungene Verknüpfung von Theorie und Empirie
}

Egle, Christoph: Reformpolitik in Deutschland und Frankreich. Wirtschafts- und Sozialpolitik bürgerlicher und sozialdemokratischer Regierungen, VS Verlag für Sozialwissenschaften, Wiesbaden 2009, 348 Seiten, $€ 49,90$.

Christoph Egle hat sich für seine Dissertation ein sehr anspruchvolles Thema gewählt. Er untersucht Reformpolitik auf den Feldern der Fiskal-, Arbeitsmarkt und Sozialpolitik in einem doppelten Vergleich: Für den Zeitraum von 1993/94 bis 2005 vergleicht er die wichtigsten Entscheidungen bürgerlicher und sozialdemokratischer Regierungen auf den genannten Feldern in Frankreich und Deutschland. Ein Regierungswechsel hin zu einer sozialdemokratisch geführten Linksregierung in beiden Ländern 1997 beziehungsweise 1998 ermöglicht diese Vorgehensweise.

Egle fragt, warum es den beiden seit den 1990er Jahren als „reformpolitische Problemfälle“ geltenden Ländern nur in unzureichendem Maße gelang, „angesichts der Herausforderungen des globalen ökonomischen Wettbewerbs, der Alterung der Gesellschaft und sich verändernder Familienstrukturen einen ausgebauten Wohlfahrtsstaat mit einer wettbewerbsfähigen Ökonomie“ zu vereinbaren (S. 17). Reformpolitik ist in diesem Kontext „nicht mehr geprägt von einem Ausbau, sondern vielmehr von einem Zwang zum Rückund Umbau wohlfahrtsstaatlicher Leistungen“ (S. 52). Egle schließt sich dem „Mainstream der ökonomischen und politikwissenschaftlichen Forschung und Literatur" an, der „eher der Angebotsorientierung zuneigt" (S. 24).

Welche Faktoren können für dieses Reformdefizit benannt werden? Haben die Regierungen die erforderlichen Reformen gar nicht erst angepackt? War ihre Politik den Problemen nicht angemessen? Konnten - aus welchen Gründen? - die Reformen nur eingeschränkt durchgesetzt werden, oder sind sie ganz gescheitert? Lag es an der parteipolitischen Färbung der Regierung? Lag es an Struktur und Dynamik des Parteienwettbewerbs oder an den institutionellen Eigenarten des Regierungssystems? Wären letztere ausschlaggebend, so hätte die Reformpolitik in der V. Republik, einem politischen System mit einer „hohen institutionellen Handlungskapazität" (S. 19), viel erfolgreicher sein müssen als im stark fragmentierten deutschen System mit vielen Vetospielern. Wäre die Parteiorientierung maßgebend, so wäre nicht erklärbar, dass die Regierung Schröder (nach 2002) eine entschiedenere Reformpolitik betrieben hat als die bürgerlichen Regierungen beiderseits des Rheins. Egle vermutet, dass wichtige Einflussfaktoren auf der Akteursebene zu suchen sind.

Konsequenterweise greift er auf den theoretischen Ansatz des akteurszentrierten Institutionalismus zurück, der in den zentralen empirischen Kapiteln 4 und 5 getestet wird. Anhand von sechs Thesen soll die unterschiedliche Reformbilanz der verschiedenen Regierungen erklärt werden. Die parteipolitischen und die institutionellen Faktoren werden mit der Parteiendifferenzthese (Do parties matter?) beziehungsweise (in Anlehnung an Tsebelis' Vetospieler-Theorie) der Kapazitätsthese erfasst, wobei zu beiden auch eine „revisionistische“ Variante formuliert wird. In den empirischen Kapiteln (S. 89 - 288) untersucht Egle auf einer breiten Daten- und Literaturbasis recht detailliert die Politik der verschiedenen Regierungen auf den genannten Politikfeldern. 54 (!) Tabellen und 40 (!) Schaubilder visualisieren die Ausführungen. Ein Fazit zu jedem Unterkapitel ermöglicht es auch dem eiligen Leser, dem Argumentationsgang zu folgen. 
Es überrascht nicht, dass die bürgerlichen Regierungen eine weitergehende, im Kern angebotsorientierte Reformpolitik unternommen haben als die sozialdemokratischen von Lionel Jospin und Gerhard Schröder (nur bis 2002). Insofern wird die Parteiendifferenzthese bestätigt. Sie kann aber nicht die recht unterschiedliche Reformbereitschaft und Durchsetzungsfähigkeit der Regierungen Édouard Balladur, Alain Juppé und Jean-Pierre Raffarin erklären und vor allem nicht den von der Regierung Schröder nach 2002 eingeschlagenen Reformkurs. Auch die Kapazitätsthese, die den Einfluss des Regierungssystems als entscheidenden Faktor sieht, greift hier nicht. Egle erweitert die bisherigen, vor allem institutionellen Ansätze durch die Einbeziehung der Handlungsstrategie und bietet einen auf den ersten Blick paradox erscheinenden Erklärungsversuch an: Unpopuläre Reformen können leichter durchgesetzt werden, wenn es der Regierung gelingt, eine heterogene Koalition zu bilden, also die politische Opposition, zumindest Teile von ihr (in Deutschland der von ihr beherrschte Bundesrat) oder Gewerkschaften (in Frankreich) durch eine „inklusive Strategie“ einzubinden. Unilateral, mit einer „exklusiven Strategie“ verfolgte Reformen scheitern an einem mächtigen Vetospieler (Bundesrat) oder an einem breiten gewerkschaftlich-gesellschaftlichen Widerstand, selbst in einem zentralisierten Regierungssystem (vor allem die Regierung Juppé mit der Rentenreform). Die verbreitete Annahme, Mehrheitsdemokratien seien tendenziell reformfähiger als Konsensdemokratien, müsse also korrigiert werden. Allerdings verspricht eine „inklusive Strategie“ nur dann Erfolg, wenn die Reformvorhaben gemäßigt und nicht radikal sind. Das Schicksal der Rentenreformpläne von Präsident Nicolas Sarkozy wird ein neuer Test für die Stichhaltigkeit von Egles Analyse sein.

Die Reformfähigkeit sozialdemokratischer Regierungen hängt jedoch nicht in erster Linie davon ab, ob die bürgerliche Opposition, deren Reformabsichten sich mit denen der Regierung teilweise decken, in eine „heterogene Koalition“ eingebaut werden kann, sondern ob es den linken Regierungen gelingt, die sozialdemokratischen Parteien beziehungsweise deren Koalitionspartner (besonders in Frankreich) für ihre Reformen zu gewinnen. Auch für Schröders Agenda-Politik war dieses schwierige, nur mit Hilfe von Rücktrittsdrohungen durchsetzbare Unterfangen entscheidend. Ob eine SPD- oder eine PS-geführte Regierung nach den an den Wahlurnen von der SPD gemachten Erfahrungen noch einmal eine ähnliche Reformpolitik unternehmen wird, ist zweifelhaft.

Man muss nicht die Prämisse über den angeblich alternativlosen Rück- und Umbau des Wohlfahrtsstaates durch eine angebotsorientierte Politik teilen, und man muss sich auch nicht alle Schlussfolgerungen, die Egle aus seinen Analysen zieht, zu eigen machen, um die herausragende Qualität seiner Arbeit zu würdigen. Sie besticht durch ein ausgeprägtes Methodenbewusstsein, durch eine immer noch eher seltene, überzeugend gelungene Verknüpfung von Theorie und Empirie, durch einen klaren, schlüssigen Aufbau und durch eine stringente und nachvollziehbare Argumentation sowie die Verarbeitung umfangreicher Materialien und eine breite Literaturkenntnis. Auch wenn man berücksichtigt, dass der Verfasser schon mehrere einschlägige Aufsätze als Vorarbeiten veröffentlicht hat, handelt es sich bei dieser Dissertation um eine außergewöhnliche wissenschaftliche Leistung, die das übliche Niveau einer Erstlingsarbeit weit überragt.

Adolf Kimmel 